\title{
Maximization of Correct Decision Probability via Channel Switching over Rayleigh Fading Channels
}

\author{
Musa Furkan Keskin, Mehmet Necip Kurt, Mehmet Emin Tutay, Sinan Gezici, and Orhan Arikan \\ Department of Electrical and Electronics Engineering, Bilkent University, Bilkent, Ankara, 06800, Turkey \\ \{keskin, mnkurt, tutay, gezici, oarikan\}@ee.bilkent.edu.tr
}

\begin{abstract}
In this study, optimal channel switching (time sharing) strategies are investigated under average power and cost constraints in order to maximize the average number of correctly received symbols between a transmitter and a receiver that are connected via multiple additive Gaussian noise channels. The optimal strategy is shown to perform channel switching either among at most three channels with full channel utilization (i.e., no idle periods), or between at most two channels with partial channel utilization. In addition, it is stated that the optimal solution must operate at the maximum average power and the maximum average cost, which facilitates low-complexity approaches for calculating the optimal strategy. For two-channel strategies, an upper bound in terms of the noise standard deviations of the employed channels is provided for the ratio between the optimal power levels. Furthermore, a simple condition depending solely on the systems parameters is derived, under which partial channel utilization cannot be optimal. Numerical examples are presented to demonstrate the validity of the theoretical results.

Index Terms- Time sharing, channel switching, Gaussian channel, probability of correct decision, partial transmission.
\end{abstract}

\section{INTRODUCTION}

Time sharing (randomization) has attracted a great deal of interest in the literature due to its capability to provide performance improvements for communication systems [1]-[10]. In [3], it is demonstrated that the average probability of error over additive noise channels with arbitrary noise probability density functions (PDFs) can be reduced by employing optimal stochastic signaling, which performs time sharing among at most three different signal levels for each information symbol. The study in [4] investigates performance gains that can be obtained by detector randomization and stochastic signaling, and proves that the optimal receiver design is represented by time sharing (randomization) between at most two maximum a-posteriori probability (MAP) detectors corresponding to two deterministic signal vectors. [7] performs joint optimization of signal amplitudes, detectors and detector randomization factors for the downlink of a multiuser communications system. Similarly, jamming performance of average power constrained jammers can be enhanced via time sharing among different power levels [2], [5], [6]. In [2], the optimal time sharing strategy for a jammer operating over channels with symmetric unimodal noise densities is shown to correspond to on-off jamming when the average power constraint is below a certain threshold. The optimum jamming strategy that minimizes the probability of detection in the Neyman-Pearson framework is considered in [6], where it is proved that power randomization between at most two different power levels can result in the highest jamming performance over an additive noise channel with a generic PDF.

Performance enhancements via time sharing can also be observed in communication systems where the transmitter and the receiver are connected through multiple channels [2], [9]-[12]. In this case, channel switching is performed

This research was supported in part by the Distinguished Young Scientist Award of Turkish Academy of Sciences (TUBA-GEBIP 2013). by transmitting over a channel during a certain period of time and switching to another channel during the next period. In [2], the optimal channel switching strategy is considered for minimizing the average probability of error over a set of channels with additive unimodal noise under an average power constraint, and it is shown that the optimum performance can be achieved via time sharing between at most two channels and power levels. An average power constrained $M$-ary communication system with multiple additive noise channels having generic noise PDFs is studied in [10] in the context of minimizing the average probability of error by joint optimization of channel switching, stochastic signaling, and detection strategies. It is demonstrated that the optimal strategy is to employ deterministic signaling or time sharing between at most two signal constellations over a single channel, or channel switching between two channels with deterministic signaling. The advantages of channel switching are investigated in [12] for additive Gaussian noise channels under average and peak power constraints, where the objective is to maximize average channel capacity. It is proved that the optimal solution performs channel switching between at most two different channels. [9] formulates the channel switching problem by incorporating channel costs associated with the usage of each channel for transmission and imposing an average cost constraint. The optimal channel switching strategy over a set of Gaussian channels under average power and cost constraints is shown to perform time sharing among at most three different channels.

In the previous studies, the objective functions in the context of channel switching are average probability of error [2], [9], [10] and average channel capacity [12], and it is assumed that channels are fully utilized; i.e., there always exists transmission over one of the channels and there are no idle periods. In this study, we investigate the problem of channel switching for maximizing the average number of correctly received symbols in the absence of the full transmission/utilization constraint. More specifically, we design optimal channel switching strategies over a set of Rayleigh fading channels under average power and cost constraints for the maximization of the average number of correctly received symbols. Instead of forcing full utilization of channels (i.e., no idle periods) as in [9], a more general formulation is developed for channel switching, where communication may not occur during a certain period of time, which, in some scenarios, is shown to attain a higher average probability of correct decision than full channel utilization. In addition, unlike the no fading assumption in [9], Rayleigh fading channels are considered in designing the optimal channel switching strategies. It is demonstrated that the optimum performance is achieved by channel switching either among at most three channels with full transmission or between at most two channels with partial transmission (Proposition 1). Also, theoretical results are obtained for characterizing the optimality of various channel switching strategies (Proposition 2 and Proposition 3), and conditions for 
the optimality of full data transmission are derived in terms of channel costs, standard deviations of channel noise and channel fading statistics (Proposition 4). Numerical examples are presented for the illustration of theoretical results.

\section{System Model And Problem Formulation}

The channel switching problem is formulated for an $M$ ary communication system with $K$ Rayleigh fading channels between the transmitter and the receiver, as illustrated in Fig. 1. Channels are assumed to be frequency non-selective to eliminate intersymbol interference and block fading to ensure constant fading coefficient over a number of symbols. In order to enhance system performance, the transmitter performs channel switching among $K$ channels over time in perfect synchronization with the receiver, that is, time sharing is performed among different block fading channels by using only one channel in a certain fraction of time [2], [10]. Fraction of time during which transmission is performed over channel $i$ is denoted by $\lambda_{i}$, which is called the channel switching factor for channel $i$. The channel switching factors satisfy $\sum_{i=1}^{K} \lambda_{i} \leq$ 1 and $\lambda_{i} \geq 0, \forall i \in\{1 \ldots K\}$. Thus, unlike the previous studies such as [2], [9], [10], it is possible to have idle periods of communications where symbol transmission/reception is not performed (in the case of $\sum_{i=1}^{K} \lambda_{i}<1$ ), which can provide performance improvements under certain conditions as compared to full utilization of channels.

Generic $M$-ary modulation is considered for communication over each channel. The received signal corresponding to the $i$ th Rayleigh fading channel can be expressed as

$$
y=\sqrt{P_{i}} \alpha_{i} s_{i}^{(j)}+n_{i}
$$

for $j \in\{0,1, \ldots, M-1\}$ and $i \in\{1, \ldots, K\}$, where $s_{i}^{(0)}, s_{i}^{(1)}, \ldots, s_{i}^{(M-1)}$ denote the set of transmitted signals (with unit average energy) employed for $M$-ary communications over channel $i, P_{i}$ determines the average power of the transmitted signal for channel $i, \alpha_{i}$ is the complex fading coefficient of the $i$ th channel, and $n_{i}$ is circularly-symmetric complex Gaussian noise over channel $i$ with mean zero and variance $2 \sigma_{i}^{2}$. It is assumed that the noise components are independent across the channels and they are also independent of the fading coefficients and the transmitted signals. In addition, equally likely symbols are assumed so that the prior probability of each symbol $s_{i}^{(j)}$ for $j \in\{0,1, \ldots, M-1\}$ is equal to $1 / M$. The fading coefficient $\alpha_{i}$ for channel $i$ is modeled as a zero-mean, circularly-symmetric complex Gaussian random variable with variance $\varsigma_{i}^{2}$ (which corresponds to Rayleigh fading). It is assumed that the receiver has the channel state information; that is, $\alpha_{i}$ is perfectly estimated at the receiver. The signal-to-noise ratio (SNR) per symbol is defined as

$$
\gamma_{i}=P_{i}\left|\alpha_{i}\right|^{2} /\left(2 \sigma_{i}^{2}\right) \cdot
$$

For coherent demodulation, the generic expression for the probability of symbol error corresponding to the SNR in (2) over Gaussian channels can be expressed exactly or approximately (depending on the modulation type and order) as [13]

$$
\mathrm{P}_{s}\left(\gamma_{i}\right)=\eta Q\left(\kappa \sqrt{\gamma_{i}}\right)
$$

where $\eta$ and $\kappa$ are constant parameters that depend on the modulation type and order. For the Rayleigh fading model described in the previous paragraph, $\gamma_{i}$ in (2) becomes an exponential random variable, and the average probability of

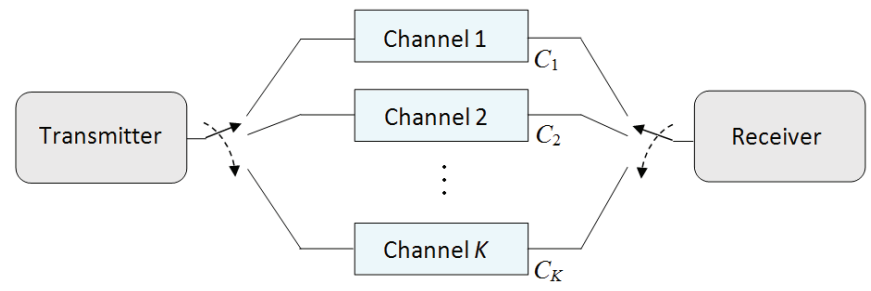

Fig. 1. Channel switching among $K$ Rayleigh fading, additive Gaussian noise channels, where $C_{i}$ denotes the cost of using channel $i$.

symbol error can be obtained by calculating the expected value of (3) over that exponential distribution, which yields [13]

$$
g_{i}(P)=\tilde{\eta}\left(1-\sqrt{\frac{\tilde{\kappa} P}{\tilde{\kappa} P+\sigma_{i}^{2} / \varsigma_{i}^{2}}}\right)
$$

where $g_{i}(P)$ represents the average probability of symbol error over channel $i$ for a power level of $P, \tilde{\eta} \triangleq \eta / 2$ and $\tilde{\kappa} \triangleq \kappa^{2} / 2$. It is noted that $g_{i}(P)$ is a convex and monotone decreasing function of $P$ for $P \geq 0$.

In the considered system model in Fig. 1, there exist $K$ channels between the transmitter and the receiver. Each channel has a cost value, denoted by $C_{i}$ for $i \in\{1, \ldots, K\}$, which represents the cost of utilizing a channel per unit time [9], [14], [15]. Cost values are nonnegative, and the relation between costs of different channels is given by $C_{i}>C_{j}$ if $\varsigma_{i}^{2} / \sigma_{i}^{2}>\varsigma_{j}^{2} / \sigma_{j}^{2}, \forall j \neq i$. This is motivated by the fact that a channel with a higher $\varsigma_{i}^{2} / \sigma_{i}^{2}$ value (equivalently, higher SNR) yields a lower average probability of symbol error as suggested by (4), which requires such a channel to have a higher cost [15], [16].

This study aims to perform the joint optimization of channel switching factors and signal powers (under average power and cost constraints) in order to maximize the average number of correctly received symbols, which is expressed as $T R \sum_{i=1}^{K} \lambda_{i} \mathrm{P}_{\mathrm{c}, i}$, where $T$ is the duration of the communication interval (during which channel switching is performed), $R$ is the symbol rate of each channel, $\mathrm{P}_{\mathrm{c}, i}$ denotes the average probability of correct decision over channel $i$ for a power level of $P_{i}$, and $\lambda_{i}$ is the channel switching factor. Considering given (fixed) values for $T$ and $R$, the aim becomes the maximization of $\sum_{i=1}^{K} \lambda_{i} \mathrm{P}_{\mathrm{c}, i}$, which will be referred to as the "average probability of correct decision" in the remainder of this study (even though $\lambda_{i}$ 's do not always add up to one). The average probability of correct decision can be stated as

$$
\sum_{i=1}^{K} \lambda_{i} \mathrm{P}_{\mathrm{c}, i}=\sum_{i=1}^{K} \lambda_{i}\left(1-g_{i}\left(P_{i}\right)\right) \triangleq \sum_{i=1}^{K} \lambda_{i} h_{i}\left(P_{i}\right)
$$

where $h_{i}\left(P_{i}\right)$ represents the average probability of correct decision over channel $i$ for a power level of $P_{i}$, which is calculated as

$$
\mathrm{P}_{\mathrm{c}, i}=1-g_{i}\left(P_{i}\right) \triangleq h_{i}\left(P_{i}\right)
$$

with $g_{i}\left(P_{i}\right)$ denoting the average probability of symbol error as computed in (4).

In the considered scenario, channel switching is performed over a certain communication interval that consists of a large number of symbols. It is assumed that the statistics of the fading coefficients, $\varsigma_{i}^{2}$ 's, are fixed in the communication interval but the fading coefficients change from block to block independently, where each block consists of a number of symbols and the block duration is significantly shorter than the 
communication interval. Also, in the communication interval, the transmitter is assumed to have the knowledge of the $\varsigma_{i}^{2} / \sigma_{i}^{2}$ term for each channel but it does not know the fading coefficient for each symbol.

It is noted that for any two channels, the one with a higher cost always results in a higher probability of correct decision for the same power level; that is, if $C_{i}>C_{j}$ (which implies $\varsigma_{i}^{2} / \sigma_{i}^{2}>\varsigma_{j}^{2} / \sigma_{j}^{2}$ ), then $h_{i}(P)>h_{j}(P)$ for all $P>0$ (cf. (4) and (5)). Several constraints must be imposed while maximizing the average probability of correct decision in order for the channel switching strategies to be applicable in practical settings. Namely, there exists an average power constraint, which can be stated as $\sum_{i=1}^{K} \lambda_{i} P_{i} \leq A_{\mathrm{p}}$, where $A_{\mathrm{p}}$ represents the average power limit. Also, an average transmission cost constraint can be expressed as $\sum_{i=1}^{K} \lambda_{i} C_{i} \leq$ $A_{\mathrm{c}}$, where $A_{\mathrm{c}}$ denotes the average cost limit [9]. Then, the following optimization problem is considered:

$$
\begin{aligned}
\max _{\left\{\lambda_{i}, P_{i}\right\}_{i=1}^{K}} & \sum_{i=1}^{K} \lambda_{i} h_{i}\left(P_{i}\right) \\
\text { subject to } & \sum_{i=1}^{K} \lambda_{i} P_{i} \leq A_{\mathrm{p}}, \quad \sum_{i=1}^{K} \lambda_{i} C_{i} \leq A_{\mathrm{c}}, \\
& \sum_{i=1}^{K} \lambda_{i} \leq 1, \quad \lambda_{i} \geq 0, \quad \forall i \in\{1 \ldots K\} .
\end{aligned}
$$

The optimization problem in (7) searches over both full transmission strategies (i.e., $\sum_{i=1}^{K} \lambda_{i}=1$ ) and partial transmission strategies (i.e., $\sum_{i=1}^{K} \lambda_{i}<1$ ) in order to achieve the maximum average probability of correct decision under average power and cost constraints.

In the remainder of the study, it is assumed without loss of generality that the ratio between the variances of the noise and the Rayleigh fading coefficient, $\sigma_{i}^{2} / \varsigma_{i}^{2}$, is distinct for each channel. This is based on the fact that if there are multiple channels with the same ratio of variances, channel switching between such channels can never increase the average probability of correct decision compared to employing only one of them at the same average power for the total duration of time, which is due to the concavity of the average probability of correct decision expressions, $h_{i}(\cdot)$. For this reason, the problem formulation that considers only the channels with distinct ratios of variances is sufficient to obtain the overall optimal solution.

\section{Optimal Channel Switching}

In this section, the optimal channel switching problem in (7) is examined in detail. In particular, the problem in (7) is reduced to a simpler form and the optimal strategies are obtained based on low-complexity calculations. The assumption made about the ordering of channel costs without loss of generality is that the cost values satisfy $C_{1}>C_{2}>\cdots>C_{K}$, thus the ratios of variances are ordered as $\varsigma_{1}^{2} / \sigma_{1}^{2}>\varsigma_{2}^{2} / \sigma_{2}^{2}>$ $\cdots>\varsigma_{K}^{2} / \sigma_{K}^{2}$. In this case, the probabilities of correct decision satisfy $h_{1}(P)>h_{2}(P)>\cdots>h_{K}(P)$ for all $P \geq 0$.

Based on the ordering of channel costs, it is clear that if $A_{\mathrm{c}} \geq C_{1}$, the optimal solution of (7) is to transmit over channel 1 exclusively with power $A_{\mathrm{p}}$. Since transmission over channel 1 results in the highest average probability of correct decision among all the channels, the optimal approach becomes the use of the best channel (channel 1) all the time at the maximum power limit when the budget allows it.
In the remainder of the study, the case of $A_{\mathrm{c}}<C_{1}$ is considered. It is straightforward to show that the solution of the problem in (7) always satisfies the average power constraint with equality since $h_{i}(P)$ is a monotone increasing function of $P$ for all $i \in\{1, \ldots, K\}$. Mathematically speaking, if $\left\{\lambda_{i}^{*}, P_{i}^{*}\right\}_{i=1}^{K}$ denotes the solution of the optimization problem in (7), then $\sum_{i=1}^{K} \lambda_{i}^{*} P_{i}^{*}=A_{\mathrm{p}}$. Furthermore, based on a similar approach to the proof of Proposition 1 in [9] with slight modifications to consider the partial transmission strategies, it can be inferred that the optimal channel switching solution operates at the average cost limit, that is, $\sum_{i=1}^{K} \lambda_{i}^{*} C_{i}^{*}=A_{\mathrm{c}}$. Hence, an optimal channel switching strategy must utilize all the available average power and average cost for $A_{\mathrm{c}}<C_{1}$. Therefore, the optimization problem in (7) can be solved by considering equality constraints (instead of inequality constraints) for the average power and average cost, which provides an important reduction in computational complexity.

In the following proposition, it is stated that the optimal channel switching strategy, which is obtained as the solution of (7), corresponds to channel switching either among at most $\min \{K, 3\}$ channels with full transmission or between at most $\min \{K, 2\}$ channels with partial transmission. (The proofs of the propositions are not presented due to the space limitation.)

Proposition 1: Assume that the power levels satisfy $P_{i} \in$ $\left[0, P_{\max }\right]$ for some finite $P_{\max }$. Then, the optimal channel switching strategy is to switch either among at most $\min \{K, 3\}$ channels with full transmission, or to switch between at most $\min \{K, 2\}$ channels with partial transmission.

Based on Proposition 1, an optimal channel switching solution corresponds to one of the following strategies: Partial/full transmission over a single channel, partial/full transmission over two channels, and full transmission over three channels. The following sections explore the details of these strategies.

\section{A. Single Channel Strategies}

Optimal solutions for full and partial transmission over a single channel are investigated in this subsection.

Strategy 1P - Partial Transmission over a Single Channel: In this case, one of the channels is employed partially; that is, a single channel is used during the busy period, and an idle period exists, as well. A partial transmission strategy that employs a channel with a cost smaller than $A_{\mathrm{c}}$ cannot be optimal (i.e., the solution of (7)) since the optimal solution must operate at the average cost limit $A_{\mathrm{c}}$, as discussed above (the third paragraph of Section III).

In some cases, the optimal channel switching strategy corresponds to Strategy 1P. In those scenarios, the optimal solution must be searched among channels with costs higher than $A_{\mathrm{c}}$. Let $\mathcal{S}_{g} \triangleq\left\{l \in\{1, \ldots, K\}: C_{l}>A_{\mathrm{c}}\right\}$. Assume that channel $i \in \mathcal{S}_{g}$ is employed with channel switching factor $\lambda_{i}$ and power $P_{i}$. Then, $\lambda_{i} P_{i}=A_{\mathrm{p}}$ and $\lambda_{i} C_{i}=A_{\mathrm{c}}$. Therefore, the optimal solution for channel $i$ is obtained as $\lambda_{i}^{*}=A_{\mathrm{c}} / C_{i}$ and $P_{i}^{*}=A_{\mathrm{p}} C_{i} / A_{\mathrm{c}}$. Hence, the probability of correct decision for channel $i$ is given by

$$
\lambda_{i}^{*} h_{i}\left(P_{i}^{*}\right)=\frac{A_{\mathrm{c}}}{C_{i}} h_{i}\left(A_{\mathrm{p}} \frac{C_{i}}{A_{\mathrm{c}}}\right)
$$

and the channel that yields the optimal solution under Strategy $1 \mathrm{P}$ is obtained as

$$
i^{*}=\underset{i \in \mathcal{S}_{g}}{\arg \max } \frac{A_{\mathrm{c}}}{C_{i}} h_{i}\left(A_{\mathrm{p}} \frac{C_{i}}{A_{\mathrm{c}}}\right) .
$$


Strategy 1F - Full Transmission over a Single Channel: In this case, one of the channels is employed all the time. This strategy may be the optimal channel switching strategy if there exists a channel with cost $A_{\mathrm{c}}$ since otherwise the average cost cannot be equal to $A_{\mathrm{c}}$.

\section{B. Two-Channel Strategies}

There exist two strategies for channel switching between two channels: Partial transmission over two channels and full transmission over two channels.

Strategy 2P - Channel Switching between Two Channels with Partial Transmission: In this strategy, channel switching is performed between two different channels and the sum of the channel switching factors is smaller than 1 , i.e., there exists an idle period with no data transmission. Let channel $i$ and channel $j$ denote the channels employed in this strategy. Then, the problem in (7) can be formulated under Strategy $2 \mathrm{P}$ as

$$
\begin{aligned}
\max _{\lambda_{i}, \lambda_{j}, P_{i}, P_{j}} & \lambda_{i} h_{i}\left(P_{i}\right)+\lambda_{j} h_{j}\left(P_{j}\right) \\
\text { subject to } & \lambda_{i} P_{i}+\lambda_{j} P_{j}=A_{\mathrm{p}}, \\
& \lambda_{i} C_{i}+\lambda_{j} C_{j}=A_{\mathrm{c}}, \\
& \lambda_{i}+\lambda_{j}<1, \quad \lambda_{i}, \lambda_{j} \in[0,1) .
\end{aligned}
$$

It is noted that Strategy $1 \mathrm{P}$ is covered as a special case of Strategy 2P. It is observed from the average cost constraint in (10) that, for the optimal channel switching between two channels, at least one of the channels should have a cost higher than $A_{\mathrm{c}}$. Therefore, to obtain the optimal solution for Strategy 2P, the problem in (10) should be solved for $K_{\mathrm{g}}(K-1)$ channel pairs, where $K_{\mathrm{g}}$ is the number of channels the costs of which are higher than $A_{\mathrm{c}}$ and $K$ is the total number of channels.

Based on the argument in the previous paragraph, assume, without loss of generality, that $C_{i}>C_{j}$ for the problem in (10). Using the average power and cost constraints in (10), the optimal value of $\lambda_{j}$ and $P_{j}$ can be expressed in terms of the optimal values of $\lambda_{i}$ and $P_{i}$ as $\lambda_{j}=\left(A_{\mathrm{c}}-\lambda_{i} C_{i}\right) / C_{j}$ and $P_{j}=\left(A_{\mathrm{p}}-\lambda_{i} P_{i}\right) / \lambda_{j}$. Therefore, the optimization problem in (10) can be simplified significantly by optimizing over two variables instead of four variables by using the two equality constraints. In addition, by considering the definition of function $h_{i}$, that is, $h_{i}(P)=1-g_{i}(P)$, where $g_{i}(P)$ is defined in (4), the optimization problem in (10) can be expressed as follows:

$$
\begin{aligned}
& \max _{\substack{\lambda_{i} \in\left[0, A_{\mathrm{c}} / C_{i}\right) \\
P_{i} \in\left[0, A_{\mathrm{p}} / \lambda_{i}\right]}}(1-\tilde{\eta})\left(\frac{A_{\mathrm{c}}-\lambda_{i} C_{i}}{C_{j}}\right) \\
& +\tilde{\eta}\left(\lambda_{i} \sqrt{\frac{\tilde{\kappa}}{\tilde{\kappa}+\frac{\sigma_{i}^{2}}{\varsigma_{i}^{2} P_{i}}}}+\frac{A_{\mathrm{c}}-\lambda_{i} C_{i}}{C_{j}} \sqrt{\frac{\tilde{\kappa}}{\tilde{\kappa}+\frac{\sigma_{j}^{2}\left(A_{\mathrm{c}}-\lambda_{i} C_{i}\right)}{\varsigma_{j}^{2}\left(A_{\mathrm{p}}-\lambda_{i} P_{i}\right) C_{j}}}}\right)
\end{aligned}
$$

where the constraints for $\lambda_{i}$ and $P_{i}$ are obtained from the relations $\lambda_{i} C_{i}+\lambda_{j} C_{j}=A_{\mathrm{c}}$ and $\lambda_{i} P_{i}+\lambda_{j} P_{j}=A_{\mathrm{p}}$. From (11), it is observed that the optimal solution for Strategy $2 \mathrm{P}$ requires a search over a two-dimensional space only (for each possible channel pair). This two-dimensional search must be executed by first determining a value for $\lambda_{i}$ and then finding the optimal $P_{i}$ for the current $\lambda_{i}$ value since the search interval for $P_{i}$ depends on the value of $\lambda_{i}$. Finally, the maximum for all those $\left(\lambda_{i}, P_{i}\right)$ pairs is calculated and the pair that yields the maximum value of the objective function is determined to be optimal.
Strategy 2F - Channel Switching between Two Channels with Full Transmission: In this strategy, channel switching is performed between two different channels and the sum of channel switching factors is equal to 1 . The formulation of the problem in (7) under Strategy $2 \mathrm{~F}$ is the same as (10) except for the constraint related to the sum of the channel switching factors, which, under Strategy $2 \mathrm{~F}$, must be $\lambda_{i}+\lambda_{j}=1$ and $\lambda_{i}, \lambda_{j} \in[0,1]$. In this case, the optimization can be performed over a single variable. Strategy $2 \mathrm{~F}$ reduces to Strategy $1 \mathrm{~F}$ if one of the channel switching factors is equal to 1 .

The following proposition introduces an upper bound for the ratio between the optimal power levels obtained for Strategy $2 \mathrm{P}$ and Strategy $2 \mathrm{~F}$.

Proposition 2: Let the solution of the optimization problem in (7) under the two-channel strategies be denoted by $\left\{\lambda_{i}^{*}, P_{i}^{*}, \lambda_{j}^{*}, P_{j}^{*}\right\}$ and suppose that $\lambda_{i}^{*}>0, \lambda_{j}^{*}>0$ and $C_{i}>C_{j}$. Then, the ratio between the optimal power levels is upper bounded by $\varsigma_{i}^{2} \sigma_{j}^{2} /\left(\varsigma_{j}^{2} \sigma_{i}^{2}\right)$.

Based on Proposition 2, it is deduced that the ratio between the optimal power levels cannot be larger than the ratio between the $\varsigma_{i}^{2} / \sigma_{i}^{2}$ and $\varsigma_{j}^{2} / \sigma_{j}^{2}$ terms, which are related to the SNRs of the channels.

\section{Three-Channel Strategies}

Based on Proposition 1, there exists only one strategy for channel switching among three channels.

Strategy 3 - Channel Switching among Three Channels: In this strategy, transmission is performed by switching among three different channels and the channel switching factors add up to 1 (i.e., full transmission).

\section{Comparison of Channel Switching Strategies}

Once the optimal solutions for the possible strategies are found, the average probabilities of correct decision corresponding to those solutions can be compared to determine the overall optimal strategy.

Firstly, the single channel strategies are examined in the context of average probability of correct decision comparison to put forward a suboptimal solution when only a single channel is employed. Conditions are investigated under which full or partial transmission over a single channel (Strategy 1P or Strategy 1F) is optimal. Strategy $1 \mathrm{~F}$ can be optimal only if there exists a channel with cost $A_{\mathrm{c}}$; otherwise, the cost budget would be used partially and the solution would not be optimal. Hence, the comparison of Strategy $1 \mathrm{~F}$ versus Strategy $1 \mathrm{P}$ as candidates for the overall optimal solution can be made as follows:

$$
h_{i^{*}}\left(A_{\mathrm{p}}\right) \gtreqless \max _{j \in \mathcal{S}_{g}} \frac{A_{\mathrm{c}}}{C_{j}} h_{j}\left(A_{\mathrm{p}} \frac{C_{j}}{A_{\mathrm{c}}}\right)
$$

where $\mathcal{S}_{g} \triangleq\left\{l \in\{1, \ldots, K\}: C_{l}>A_{\mathrm{c}}\right\}, i^{*}$ is the index of the channel satisfying $C_{i^{*}}=A_{\mathrm{c}}$, and the left-hand-side and the right-hand-side of (12) represent the average probabilities of correct decision for the optimal solutions of Strategy $1 \mathrm{~F}$ and Strategy $1 \mathrm{P}$, respectively.

The following proposition presents a sufficient condition for deciding between two channels in terms of optimality under the single channel strategies, Strategy 1P or Strategy 1F.

Proposition 3: Consider a channel pair $(i, j)$ such that $C_{i}>C_{j} \geq A_{\mathrm{c}}$. If the condition

$$
\frac{\sigma_{j}^{2} / \varsigma_{j}^{2}}{\sigma_{i}^{2} / \varsigma_{i}^{2}} \leq \frac{1-\frac{C_{j}}{C_{i}}}{\tilde{\eta}}+\frac{C_{j}}{C_{i}}
$$


is satisfied, then partial/full transmission over channel $j$ achieves a higher average probability of correct decision than partial transmission over channel $i$.

A simpler condition which does not involve the calculations of $g_{i}\left(\right.$ or, $\left.h_{i}\right)$ is provided in Proposition 3 as compared to (9) and (12) for determining whether Strategy 1F achieves a higher average probability of correct decision than Strategy 1P if there exists a channel with cost $A_{\mathrm{c}}$, and for deciding between two channels in terms of average probability of correct decision under Strategy 1P otherwise. The inverse of Proposition 3 may not be valid as it puts forward only a sufficient condition for deciding between the two cases. As a reasonable approach, the condition in (13) can be checked first, and if it is not satisfied, then the necessary and sufficient conditions in (8) and (12) can be examined. Proposition 3 can especially be useful for applications where the system does not have sufficient time or capability (due to hardware, complexity, etc. limitations) to switch among different channels, thereby constraining themselves to use a single channel only.

One of the main results in this study is the following proposition, which presents a sufficient condition under which partial transmission (Strategy 2P or Strategy 1P) cannot be optimal. That is, it is guaranteed under the stated conditions that partial transmission over a single channel or two channels is outperformed by a full transmission strategy.

Proposition 4: Assume that there exists a channel $k \in$ $\{1, \ldots, K\}$ satisfying the conditions

$$
C_{k} \leq A_{\mathrm{c}} \text { and } \frac{\sigma_{k}^{2} / \varsigma_{k}^{2}}{\sigma_{i}^{2} / \varsigma_{i}^{2}} \leq \frac{1-\frac{C_{k}}{C_{i}}}{\tilde{\eta}}+\frac{C_{k}}{C_{i}}, \forall i \in \mathcal{S}_{g}
$$

where $\mathcal{S}_{g}=\left\{l \in\{1, \ldots, K\}: C_{l}>A_{\mathrm{c}}\right\}$. Then, partial data transmission is not optimal.

Proposition 4 is highly crucial in that it provides a condition that definitely removes the computational burden of solving the optimization problem in (10), which involves both Strategy 2P and Strategy 1P. Hence, it suffices to solve the optimization problem in Strategy 3 only in order to obtain the optimal solution of (7), thereby greatly reducing the computational complexity. In addition, the condition derived in Proposition 4 does not depend on the optimal power levels or channel switching factors; it depends only on scenario parameters such as channel costs, channel noise variances, and statistics of fading coefficients. Therefore, given a set of communication channels with assigned costs and known noise and fading statistics, if the condition in (14) is satisfied, it can be stated beforehand that partial data transmission is not optimal.

\section{NumERiCAL EXAMPLES}

In this section, the validity of the theoretical results is demonstrated via numerical examples. Comparison of the following strategies are performed in the numerical studies:

Partial Transmission: In this strategy, it is possible to have idle periods where no data transmission occurs. One or two channels should be employed for partial transmission due to Proposition 1. The optimal solutions for this approach are obtained by using Strategy $1 \mathrm{P}$ and Strategy 2P, which converges to Strategy $1 \mathrm{P}$ when one of the optimal channel switching factors equals to zero.

Full Transmission: In this strategy, there are no idle periods during transmission, and one, two or three channels are employed due to Proposition 1. Strategy 1F, Strategy 2F and Strategy 3 are employed to find the optimal solution in this case. Strategy 3 converges to Strategy $2 \mathrm{~F}$ when one of the optimal channel switching factors equals to zero, and to Strategy $1 \mathrm{~F}$ when two of the optimal channel switching factors equal to zero.

Two scenarios with different modulation types for symbol transmission are presented to explore the performance improvements that can be obtained via partial/full transmission and channel switching. In the first scenario, binary phase shift keying (BPSK) modulation is employed. For BPSK in Rayleigh fading, the parameters of the probability of correct decision $h_{i}$ are found to be $\tilde{\eta}=0.5$ and $\tilde{\kappa}=1$ (cf. (4)). Also, there exist $K$ Rayleigh fading channels, and the Gaussian noise standard deviations, the fading coefficient standard deviations, and the costs of the channels are represented, for notational simplicity, in the vector form as $\boldsymbol{\sigma}=\left[\sigma_{1} \cdots \sigma_{K}\right]$, $\varsigma=\left[\varsigma_{1} \cdots \varsigma_{K}\right]$, and $\boldsymbol{C}=\left[C_{1} \cdots C_{K}\right]$, respectively. A four channel scenario with the following parameters is studied: $\boldsymbol{\sigma}=\left[\begin{array}{llll}0.0316 & 0.15 & 20 & 25.1247\end{array}\right], \boldsymbol{\varsigma}=\left[\begin{array}{llll}1 & 1.5 & 2 & 2.5\end{array}\right], \boldsymbol{C}=$ $\left[\begin{array}{llll}4 & 1.1 & 1 & 0.95\end{array}\right]$, and the average cost limit is set to 1 ; that is, $A_{\mathrm{c}}=1$. In Fig. 2, the average probabilities of correct decision are plotted versus the average power limit $A_{\mathrm{p}}$ for the optimal solutions of the five possible strategies, namely, Strategy 1P, Strategy 1F, Strategy 2P, Strategy 2F, and Strategy 3. It is observed that the performance of the full transmission strategies is never higher than that of the partial transmission strategies for all values of $A_{\mathrm{p}}$. This is due to the fact that the optimal solution of the partial transmission strategies can converge to that of the full transmission strategies (Strategy $1 \mathrm{~F}$ and Strategy 2F) in cases where full transmission is optimal. An important observation is that the optimal partial transmission strategy outperforms the optimal full transmission strategy for $A_{\mathrm{p}} \in(0.0003,174.1)$, in which there are sub-intervals where both partial transmission over a single channel and channel switching between two channels with partial utilization can be the overall optimal strategy (Strategy $1 \mathrm{P}$ is optimal for $\left.A_{\mathrm{p}} \in(0.0007,3.848)\right)$. In addition, it is remarkable that the use of partial transmission instead of full transmission under the same average power and cost constraints is observed to increase the average probability of correct decision by as much as 22 percent. For very small and very large values of $A_{\mathrm{p}}$, all the strategies converge to each other, indicating that Strategy $1 \mathrm{~F}$ is the optimal one, which is also theoretically possible since there exists a channel with cost $A_{\mathrm{c}}$ in this scenario. The parameters of the overall optimal strategy are presented in Table I for some values of $A_{\mathrm{p}}$. In the table, the optimal channel switching solution is represented by channel switching factors $\left(\lambda_{i}, \lambda_{j}, \lambda_{k}\right)$ and power levels $\left(P_{i}, P_{j}, P_{k}\right)$, where $i<j<k$. The channels that are not employed in the optimal solution are marked with "_" in the table. Since at most three channels can be utilized in the optimal solution according to Proposition 1, only three of the channel switching factors are shown in the table. It should be noted that $\lambda_{i}$, $\lambda_{j}$, and $\lambda_{k}$ correspond to the channel switching factors of the employed channels with the smallest index, the second smallest index, and the third smallest index, respectively. For example, for $A_{\mathrm{p}}=500$, channel 2 is employed with channel switching factor 0.3333 and power 8.1563 , and channel 4 is employed with channel switching factor 0.6667 and power 745.92. Table I demonstrates that the optimal strategy may employ a single channel or two channels, and perform full or partial utilization of channels for transmission, as stated in Proposition 1. It is observed from Fig. 2 and Table I that for $A_{\mathrm{p}} \in(3.848,174.1)$, channel switching between channel 2 and channel 4 with partial utilization outperforms 


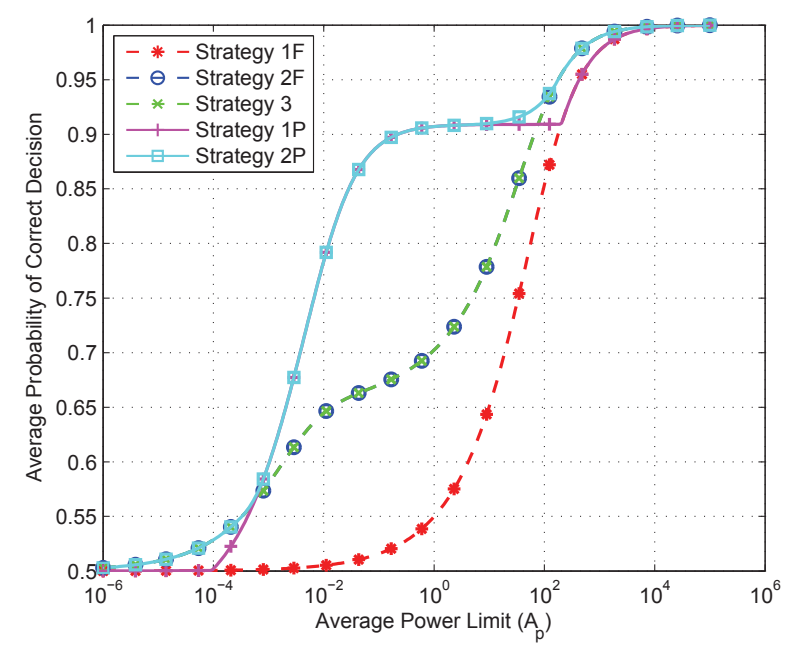

Fig. 2. Average probability of correct decision versus $A_{\mathrm{p}}$ in the first scenario.

TABLE I

Parameters of optimal Channel SWitching Strategy in Fig. 2.

\begin{tabular}{|c|ccc|cccc|}
\hline$A_{\mathrm{p}}$ & $\lambda_{i}$ & $\lambda_{j}$ & $\lambda_{k}$ & $P_{1}$ & $P_{2}$ & $P_{3}$ & $P_{4}$ \\
\hline 0.0005 & 0.5419 & 0.4251 & - & - & 0.000922 & - & 0.00000011 \\
0.001 & 0.9090 & - & - & - & 0.0011 & - & - \\
0.01 & 0.9090 & - & - & - & 0.011 & - & - \\
0.05 & 0.9090 & - & - & - & 0.055 & - & - \\
0.5 & 0.9090 & - & - & - & 0.55 & - & - \\
50 & 0.7464 & 0.1882 & - & - & 3.2287 & - & 252.75 \\
500 & 0.3333 & 0.6667 & - & - & 8.1563 & - & 745.92 \\
1000 & 0.3333 & 0.6667 & - & - & 15.588 & - & 1492.2 \\
\hline
\end{tabular}

single channel strategies Strategy 1P and Strategy 1F, which employ channel 2 and channel 3, respectively. The ratio of the optimal power levels for solutions involving two channels is calculated to confirm the validity of Proposition 2 for some values of the average power limit. For $A_{\mathrm{p}}=0.0005, P_{2} / P_{4}=$ $7749.6181<\varsigma_{2}^{2} \sigma_{4}^{2} /\left(\varsigma_{4}^{2} \sigma_{2}^{2}\right)=10100$ and for $A_{\mathrm{p}}=500$, $P_{2} / P_{4}=91.4528<\varsigma_{2}^{2} \sigma_{4}^{2} /\left(\varsigma_{4}^{2} \sigma_{2}^{2}\right)=10100$, which are in compliance with Proposition 2.

The second scenario utilizes 8-level pulse amplitude modulation (8-PAM), where the noise standard deviations, the fading coefficient standard deviations, the channel costs, and the average cost limit are given by $\sigma=$ $\left[\begin{array}{llllll}0.0141 & 0.1118 & 0.0632 & 1.2649 & 3\end{array}\right], \varsigma=\left[\begin{array}{lllll}0.1 & 0.5 & 0.2 & 0.4 & 0.3\end{array}\right]$, $\boldsymbol{C}=\left[\begin{array}{lllll}10 & 8 & 6 & 4 & 2\end{array}\right]$, and $A_{\mathrm{c}}=5$, respectively. Modulation parameters in (4) are determined to be $\tilde{\eta}=0.8750$ and $\tilde{\kappa}=$ 0.0476. Fig. 3 shows the average probability of correct symbol decision with respect to $A_{\mathrm{p}}$. Similar observations to those in the previous scenario are made. In particular, Strategy $1 \mathrm{~F}$ achieves the lowest probability of correct decision for most $A_{\mathrm{p}}$ 's whereas Strategy 2P and Strategy 1P turn out to be the optimal strategy in distinct intervals of the considered $A_{\mathrm{p}}$ region. As an example to the validity of Proposition 2, the following cases can be examined: For $A_{\mathrm{p}}=75, P_{4} / P_{3}=$ $6.75<\beta_{4} / \beta_{3}=10$ and for $A_{\mathrm{p}}=1000, P_{3} / P_{4}=9.2824<$ $\beta_{4} / \beta_{3}=10$.

\section{CONCLUSION}

Optimal channel switching strategies over Rayleigh fading additive Gaussian noise channels have been studied under average power and cost constraints in the presence of partial and full utilization of channels. It has been stated that the optimal channel switching strategy employs at most three channels in the full transmission case and at most two channels

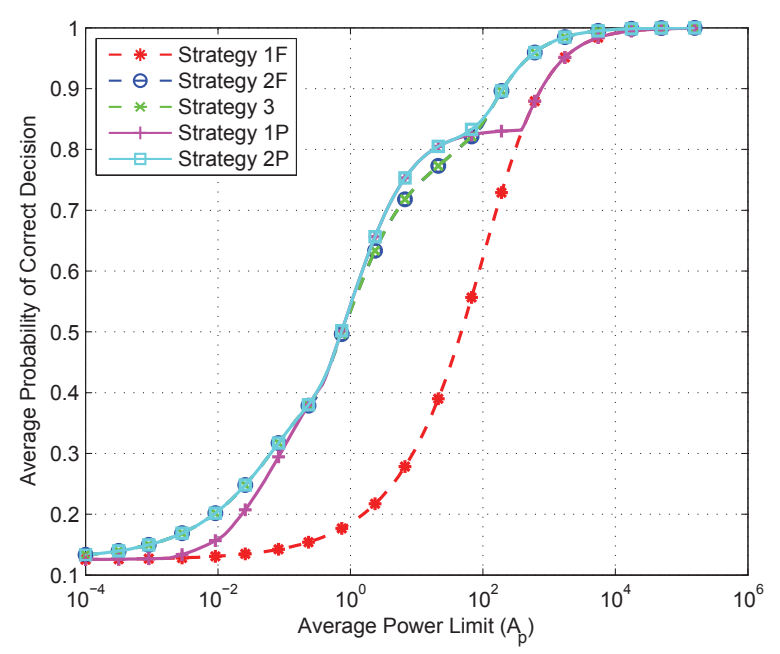

Fig. 3. Average probability of correct decision vs. $A_{\mathrm{p}}$ in the second scenario.

in the partial transmission case. In addition to characterizing the strategies that employ single or double channels, conditions that depend only on scenario parameters, namely, channel costs, noise variances, and fading statistics, have been derived under which partial data transmission cannot be optimal.

\section{REFERENCES}

[1] S. Loyka, V. Kostina, and F. Gagnon, "Error rates of the maximumlikelihood detector for arbitrary constellations: Convex/concave behavior and applications," IEEE Trans. Inform. Theory, vol. 56, no. 4, pp. 19481960, April 2010.

[2] M. Azizoglu, "Convexity properties in binary detection problems," IEEE Trans. Inform. Theory, vol. 42, no. 4, pp. 1316-1321, July 1996.

[3] C. Goken, S. Gezici, and O. Arikan, "Optimal stochastic signaling for power-constrained binary communications systems," IEEE Trans. Wireless Commun., vol. 9, no. 12, pp. 3650-3661, Dec. 2010.

[4] B. Dulek and S. Gezici, "Detector randomization and stochastic signaling for minimum probability of error receivers," IEEE Trans. Commun., vol. 60, no. 4, pp. 923-928, Apr. 2012.

[5] B. Dulek, S. Gezici, and O. Arikan, "Convexity properties of detection probability under additive Gaussian noise: Optimal signaling and jamming strategies," IEEE Trans. Signal Process., vol. 61, no. 13, pp. 3303-3310, July 2013.

[6] S. Bayram, N. D. Vanli, B. Dulek, I. Sezer, and S. Gezici, "Optimum power allocation for average power constrained jammers in the presence of non-Gaussian noise," IEEE Commun. Letters, vol. 16, no. 8, pp. 11531156, Aug. 2012.

[7] M. E. Tutay, S. Gezici, and O. Arikan, "Optimal detector randomization for multiuser communications systems," IEEE Trans. Commun., vol. 61, no. 7, pp. 2876-2889, July 2013.

[8] B. Dulek, N. D. Vanli, S. Gezici, and P. K. Varshney, "Optimum power randomization for the minimization of outage probability," IEEE Trans. Wireless Commun., vol. 12, no. 9, pp. 4627-4637, Sep. 2013.

[9] M. E. Tutay, S. Gezici, H. Soganci, and O. Arikan, "Optimal channel switching over Gaussian channels under average power and cost constraints," IEEE Trans. Commun., vol. 63, pp. 1907-1922, May 2015.

[10] B. Dulek, M. E. Tutay, S. Gezici, and O. Arikan, "Optimal signaling and detector design for M-ary communication systems in the presence of multiple additive noise channels," Digital Signal Processing, vol. 26, pp. 153-168, Mar. 2014.

[11] J. A. Ritcey and M. Azizoglu, "Performance analysis of generalized selection combining with switching constraints," IEEE Commun. Letters, vol. 4, no. 5, pp. 152-154, May 2000.

[12] A. D. Sezer, S. Gezici, and H. Inaltekin, "Optimal channel switching strategy for average capacity maximization," IEEE Trans. Commun., vol. 63, no. 6, pp. 2143-2157, June 2015.

[13] A. Goldsmith, Wireless Communications. Cambridge Univ. Press, 2005.

[14] D. Niyato and E. Hossain, "Spectrum trading in cognitive radio networks: A market-equilibrium-based approach," IEEE Wireless Commun., vol. 15 , no. 6, pp. 71-80, Dec. 2008.

[15] J. Huang, R. Berry, and M. L. Honig, "Auction-based spectrum sharing," ACM/Springer Mobile Networks and Apps., pp. 405-418, 2006.

[16] Z. Ji and K. Liu, "Dynamic spectrum sharing: A game theoretical overview," IEEE Commun. Mag., vol. 45, no. 5, pp. 88-94, May 2007. 